\title{
Diversity and Distribution of Avian Fauna of Swat, Khyber Pakhtunkhwa, Pakistan
}

\author{
Amir Jan Pathan, Shahroz Khan, Naveed Akhtar, and Kausar Saeed \\ Department of Zoology, Abdul Wali Khan University Mardan, Buner Campus, Pakistan \\ Correspondence should be addressed to Amir Jan Pathan; nghazal02@gmail.com
}

Received 18 May 2014; Revised 14 August 2014; Accepted 20 August 2014; Published 2 September 2014

Academic Editor: Luciano J. Avila

Copyright (C) 2014 Amir Jan Pathan et al. This is an open access article distributed under the Creative Commons Attribution License, which permits unrestricted use, distribution, and reproduction in any medium, provided the original work is properly cited.

\begin{abstract}
This survey was conducted from January 2013 to December 2013 to explore the avian fauna of Swat valley and to find out the major threats to the avian fauna of the area as it was neglected for years. Direct and indirect methods were used in the study by visiting the field and by interviewing the local peoples and hunters about the current and past status of the avian fauna of the area. During the current study direct and indirect methods were used. A total of 138 species were recorded belonging to 13 orders and 48 families. The order Passeriformes were recorded much in number that were 31 species. Most of the birds were migratory and few were resident. The fauna was very rich due to the flora of the area and also due to less hunting. Orders Anseriformes, Apodiformes, Charadriiformes, Columbiformes, Pelecaniformes, Phoenicopteriformes, and Psittaciformes were found migratory and orders Ciconiiformes, Coraciiformes, Galliformes, and Piciformes were found resident while some members of Gruiformes and Passeriformes were found migratory and some resident.
\end{abstract}

\section{Introduction}

Birds are one of the most popular life forms on the planet, and their diversity leads to a richness of life and beauty. Apart from this, birds have always fascinated mankind with their intrinsically beautiful plumage, melodious songs, and artistic behavior. There are around 9000 species of birds living in the world today, with a tremendous diversity of life style. Besides this, birds are valuable for many aspects; that is, they are a sensitive indicator of pollution and also play great role in pest control.

The bird species are friends of farmers who believe that bird consumes large numbers of harmful insects, as well as their eggs and larvae, which serves as a biological control agent of insect pests in Pakistan $[1,2]$.

Birds are of great economic importance to the human society. They play an important role in controlling population of different insects and pests. They play the role of scavengers and pollinating agents and also help in the dispersal of seeds of vegetation. They are helpful and help to provide rich food for mankind and are known to man since ages [3].
Wildlife management and conservation initiatives are only possible with the appropriate information on wildlife and its habitat [4]. Wildlife habitat basically comprises food, cover, and water. Each species requires a particular habitat or the space, food, shelter, and other needs of survival so much so that species are said to be the product of their habitat [5].

With regard to birds, the total number of birds species in the world today is 9040 and the total number of taxa of birds of Indo-Pakistan subcontinent is 2060 [6]. The variety of avian species in ecosystems reflects the well-being of its habitat. Birds are the indicators of environment and are being used for conservation and environmental impact assessment [7].

Of course, the Indian subcontinent, a part of the vast Oriental biogeographic regions, is very rich in biodiversity. Out of more than 9,000 birds of the world, the Indian subcontinent contains about 1,300 species, or over $13 \%$ of the world's birds [8].

Pakistan harbors a wide range of ecosystems which in turn catches the attention of a diverse avifauna to exploit their resources [9]. More than 650 species of birds have 
been reported in the country and their occurrence in three zoogeographical zones (Oriental, Palaearctic, and Ethiopian regions) is unique in the world $[10,11]$.

Although the bird is intensively hunted and captured in its native range in Pakistan, owing to which local populations could be declining, the overall status of the species is regarded as stable [12-14]. The species is a friend of farmers who believe this bird consumes large numbers of harmful insects, as well as their eggs and larvae, and serves therefore as a biological control agent of insect pests in Pakistan $[1,2]$.

The bird is normally found foraging in open cultivated tracks and grasslands intermixed with scrub forests and is rarely observed above an elevation of $1200 \mathrm{~m}$ in Pakistan [15]. The Grey Francolin (Francolinus pondicerianus) is widely associated with the drier regions of the Indus plains and has penetrated the Thar Desert in Sindh, as well as the Thal and Cholistan deserts in Punjab. The species also occurs in the lower hills of the Makran and Lasbela districts in Balochistan, the Cherat and Kohat districts of Khyber Pakhtunkhwa province, the salt range and agroforestry tracks of the Pothwar Plateau in Punjab, and the Margalla hills of Islamabad [16-18].

In Khyber Pakhtunkhwa the wild fauna is rich and its wildlife flourishing in forests is a precious heritage of the country but due to motorized and ground hunting these wildlife species were driven to a point of extinction. For this purpose it is necessary to provide the best protection to wildlife in Khyber Pakhtunkhwa; therefore several areas were declared as protected areas [19].

The present study was conducted with the following aims:

(1) to explore the avian fauna of Swat valley,

(2) to find out the major threats to the Avian fauna of the valley,

(3) to differentiate between migratory and endemic birds.

\section{Methods and Materials}

2.1. Study Area. The historic Swat Valley lies between $34^{\circ}-40^{\prime}$ to $35^{\circ} \mathrm{N}$ latitude and $72^{\prime}$ to $74^{\circ}-6^{\prime}$ E longitude and is part of the Federally Administered Tribal Areas (FATA) of the Khyber Pakhtunkhwa province of Pakistan. Total area is $5,337 \mathrm{~km}^{2}$, total population is $1,257,602$, and capital is Saidu Sharif.

The survey was conducted from January 2013 to December 2013. The data was collected by using the direct and indirect methods in order to study the present status of avian fauna of the district Swat. Direct data collection will be made by visiting the study area once or twice a day early in the morning at 8:00 am till sunset. The bird fauna were observed using binoculars and the status of each bird was stated as follows: $\mathrm{M}$ is migratory; $\mathrm{R}$ is resident; $\mathrm{C}$ is common; $\mathrm{r}$ is rare; $\mathrm{WM}$ is winter migrant; and SM is summer migrant.

In indirect data collection, hunters, wildlife staff, local residents, farmers, and other knowledgeable persons were interviewed about the present and past status of the birds diversity of the study area. The main focus was made by visiting study area rather than relaying the data which was collected indirectly.

\section{Results}

The survey was conducted from January 2013 to December 2013. In this survey total 138 species were recorded which belong to 13 orders and 48 families (see Table 1). The fauna of the study area was rich due to the current cease fire in the district Swat due to the current terrorism situation in the area. Most of the birds were migratory. Orders Anseriformes, Apodiformes, Charadriiformes, Columbiformes, Pelecaniformes, Phoenicopteriformes, and Psittaciformes were found migratory and orders Ciconiiformes, Coraciiformes, Galliformes, and Piciformes were found resident while some members of Gruiformes and Passeriformes were found migratory and some resident.

Feeding and habitat availability play an important role in the diversity and distribution of the avian fauna of area. The flora of the study area was rich and due to the thick flora the study area was bearing rich avian fauna. As the flora of the study area was very thick the Quails and Grey Partridge were found in large numbers as there were many places of shelters for their breeding and other activities.

It was observed that the birds were hunted but the hunting ratio was low due to the current situation of terrorism in Swat; therefore the bird fauna was rich. In our study the Alectoris chukar was found widely in many numbers due to less hunting in the study area due to the cease fire in Swat.

During the survey total 15 species belonging to family Anatidae were recorded and all were summer migratory (SM); all the species were rare (r) except Mergus merganser which was common. The species of family Apodidae were found migratory and were rare in number. Most of the species of the order Charadriiformes were found migratory and were summer migratory (SM) and were found to be common, while Vanellus vanellus was winter migratory (WM) and was recorded as rare (r). All species of the families Ardeidae and Ciconiidae were found resident $(\mathrm{R})$ but were rare in numbers. The species of family Columbidae were found migratory (M), and all were found as common (C) except Chalcophaps indica, Treron pompadora, and Treron phoenicoptera which were rare in numbers. The species of the order Coraciiformes were resident $(\mathrm{R})$ and were common $(\mathrm{C})$, while Upupa epops was found rare $(r)$. The members of family Phasianidae were resident $(\mathrm{R})$ and were found as common. The members of the families Turnicidae and Rallidae were resident $(R)$ and were common (C), while the members of family Gruidae were migratory $(\mathrm{M})$ and were also noted as common $(\mathrm{C})$. Most of the species of the order Passeriformes were resident (R) and were common except Sturnus vulgaris, Hirundo rustica, and Terpsiphone paradise which were found to be winter migratory (WM) and were common, while Dicrurus macrocercus and Carpodacus pulcherrimus were summer migratory (SM) and were common. All members of the order Pelecaniformes were summer migratory (SM) and were found common. Species of the order Phoenicopteriformes were found summer migratory (SM) and were rare in numbers. The Dendrocopos moluccensis was the only member of family Picidae recorded during the study and was resident (R) and was found as rare (r). Members of family Psittacidae were found migratory $(\mathrm{M})$ and were common. 
TABLE 1: Table showing the details of species recorded during the study.

\begin{tabular}{|c|c|c|c|c|}
\hline Order & Family & Scientific name & Local name & Status \\
\hline Anseriformes & Anatidae & Aythya baeri & Shingare & $\mathrm{M}(\mathrm{SM})(\mathrm{r})$ \\
\hline Anseriformes & Anatidae & Anas formosa & Shingare & $\mathrm{M}(\mathrm{SM})(\mathrm{r})$ \\
\hline Anseriformes & Anatidae & Tadorna ferruginea & Shingare & $\mathrm{M}(\mathrm{SM})(\mathrm{r})$ \\
\hline Anseriformes & Anatidae & Anas falcate & Shingare & $\mathrm{M}(\mathrm{SM})(\mathrm{r})$ \\
\hline Anseriformes & Anatidae & Anas strepera & Shingare & $\mathrm{M}(\mathrm{SM})(\mathrm{r})$ \\
\hline Anseriformes & Anatidae & Anas crecca & Shingare & $\mathrm{M}(\mathrm{SM})(\mathrm{r})$ \\
\hline Anseriformes & Anatidae & Anas platyrhynchos & Shingare & $\mathrm{M}(\mathrm{SM})(\mathrm{r})$ \\
\hline Anseriformes & Anatidae & Anas poecilorhyncha & Shingare & $\mathrm{M}(\mathrm{SM})(\mathrm{r})$ \\
\hline Anseriformes & Anatidae & Anas acuta & Shingare & $\mathrm{M}(\mathrm{SM})(\mathrm{r})$ \\
\hline Anseriformes & Anatidae & Anas clypeata & Shingare & $\mathrm{M}(\mathrm{SM})(\mathrm{r})$ \\
\hline Anseriformes & Anatidae & Aythya ferina & Shingare & $\mathrm{M}(\mathrm{SM})(\mathrm{r})$ \\
\hline Anseriformes & Anatidae & Aythya nyroca & Shingare & $\mathrm{M}(\mathrm{SM})(\mathrm{r})$ \\
\hline Anseriformes & Anatidae & Clangula hyemalis & Shingare & $\mathrm{M}(\mathrm{SM})(\mathrm{r})$ \\
\hline Anseriformes & Anatidae & Bucephala clangula & Shingare & $\mathrm{M}(\mathrm{SM})(\mathrm{r})$ \\
\hline Anseriformes & Anatidae & Mergus merganser & Shingare & $\mathrm{M}(\mathrm{SM})(\mathrm{C})$ \\
\hline Apodiformes & Apodidae & Tachymarptis melba & Lagarai & $\mathrm{M}(\mathrm{r})$ \\
\hline Apodiformes & Apodidae & Cypsiurus balasiensis & Lagarai & $\mathrm{M}(\mathrm{r})$ \\
\hline Apodiformes & Apodidae & Apus apus & Lagarai & $\mathrm{M}(\mathrm{r})$ \\
\hline Apodiformes & Apodidae & Apus pacificus & Lagarai & $\mathrm{M}(\mathrm{r})$ \\
\hline Apodiformes & Apodidae & Apus nipalensis & Lagarai & $\mathrm{M}(\mathrm{r})$ \\
\hline Apodiformes & Apodidae & Apus pallidus & Lagarai & $\mathrm{M}(\mathrm{r})$ \\
\hline Charadriiformes & Scolopacidae & Limosa lapponica & Tum Tel & $\mathrm{M}(\mathrm{SM})(\mathrm{C})$ \\
\hline Charadriiformes & Scolopacidae & Calidris ferruginea & Tum Tel & $\mathrm{M}(\mathrm{SM})(\mathrm{C})$ \\
\hline Charadriiformes & Scolopacidae & Lymnocryptes minimus & Chaghat & $\mathrm{M}(\mathrm{SM})(\mathrm{C})$ \\
\hline Charadriiformes & Scolopacidae & Calidris acuminata & Tum Tel & $\mathrm{M}(\mathrm{SM})(\mathrm{C})$ \\
\hline Charadriiformes & Scolopacidae & Limicola falcinellus & Tum Tel & $\mathrm{M}(\mathrm{SM})(\mathrm{C})$ \\
\hline Charadriiformes & Scolopacidae & Actitis hypoleucos & Tum Tel & $\mathrm{M}(\mathrm{SM})(\mathrm{C})$ \\
\hline Charadriiformes & Scolopacidae & Calidris alpina & Tum Tel & $\mathrm{M}(\mathrm{SM})(\mathrm{C})$ \\
\hline Charadriiformes & Scolopacidae & Calidris alba & Tum Tel & $\mathrm{M}(\mathrm{SM})(\mathrm{C})$ \\
\hline Charadriiformes & Scolopacidae & Calidris temminckii & Tum Tel & $\mathrm{M}(\mathrm{SM})(\mathrm{C})$ \\
\hline Charadriiformes & Dromadidae & Dromas ardeola & Tum Tel & $\mathrm{M}(\mathrm{SM})(\mathrm{C})$ \\
\hline Charadriiformes & Recurvirostridae & Himantopus himantopus & Tum Tel & $\mathrm{M}(\mathrm{SM})(\mathrm{C})$ \\
\hline Charadriiformes & Glareolidae & Cursorius cursor & Tum Tel & $\mathrm{M}(\mathrm{SM})(\mathrm{C})$ \\
\hline Charadriiformes & Glareolidae & Cursorius coromandelicus & Tum Tel & $\mathrm{M}(\mathrm{SM})(\mathrm{C})$ \\
\hline Charadriiformes & Charadriidae & Vanellus vanellus & Babozai & $\mathrm{M}(\mathrm{WM})(\mathrm{r})$ \\
\hline Charadriiformes & Charadriidae & Charadrius hiaticula & Tum Tel & $\mathrm{M}(\mathrm{SM})(\mathrm{C})$ \\
\hline Charadriiformes & Charadriidae & Vanellus leucurus & Tum Tel & $\mathrm{M}(\mathrm{SM})(\mathrm{C})$ \\
\hline Charadriiformes & Charadriidae & Charadrius mongolus & Tum Tel & $\mathrm{M}(\mathrm{SM})(\mathrm{C})$ \\
\hline Charadriiformes & Stercorariidae & Stercorarius pomarinus & Tum Tel & $\mathrm{M}(\mathrm{SM})(\mathrm{C})$ \\
\hline Charadriiformes & Laridae & Larus canus & - & $\mathrm{M}(\mathrm{SM})(\mathrm{C})$ \\
\hline Charadriiformes & Laridae & Larus heuglini & Tum Tel & $\mathrm{M}(\mathrm{SM})(\mathrm{C})$ \\
\hline Charadriiformes & Laridae & Larus ridibundus & Tum Tel & $\mathrm{M}(\mathrm{SM})(\mathrm{C})$ \\
\hline Charadriiformes & Rostratulidae & Rostratula benghalensis & Tum Tel & $\mathrm{M}(\mathrm{SM})(\mathrm{C})$ \\
\hline Charadriiformes & Haematopodidae & Haematopus ostralegus & Tum Tel & $\mathrm{M}(\mathrm{SM})(\mathrm{C})$ \\
\hline Ciconiiformes & Ardeidae & Ardea modesta & Bagh & $\mathrm{R}(\mathrm{r})$ \\
\hline Ciconiiformes & Ardeidae & Ixobrychus flavicollis & Bagh & $\mathrm{R}(\mathrm{r})$ \\
\hline Ciconiiformes & Ardeidae & Nycticorax nycticorax & Bagh & $\mathrm{R}(\mathrm{r})$ \\
\hline Ciconiiformes & Ardeidae & Ardea cinerea & Bagh & $\mathrm{R}(\mathrm{r})$ \\
\hline Ciconiiformes & Ardeidae & Ardea goliath & Bagh & $\mathrm{R}(\mathrm{r})$ \\
\hline
\end{tabular}


TABle 1: Continued.

\begin{tabular}{|c|c|c|c|c|}
\hline Order & Family & Scientific name & Local name & Status \\
\hline Ciconiiformes & Ardeidae & Ardea purpurea & Bagh & $\mathrm{R}(\mathrm{r})$ \\
\hline Ciconiiformes & Ardeidae & Egretta intermedia & Bagh & $\mathrm{R}(\mathrm{r})$ \\
\hline Ciconiiformes & Ardeidae & Egretta gularis & Bagh & $\mathrm{R}(\mathrm{r})$ \\
\hline Ciconiiformes & Ardeidae & Egretta garzetta & Bagh & $\mathrm{R}(\mathrm{r})$ \\
\hline Ciconiiformes & Ardeidae & Ardeola grayii & Bagh & $\mathrm{R}(\mathrm{r})$ \\
\hline Ciconiiformes & Ardeidae & Bubulcus ibis & Bagh & $\mathrm{R}(\mathrm{r})$ \\
\hline Ciconiiformes & Ardeidae & Butorides striata & Bagh & $\mathrm{R}(\mathrm{r})$ \\
\hline Ciconiiformes & Ardeidae & Ixobrychus minutus & Bagh & $\mathrm{R}(\mathrm{r})$ \\
\hline Ciconiiformes & Ardeidae & Ixobrychus cinnamomeus & Bagh & $\mathrm{R}(\mathrm{r})$ \\
\hline Ciconiiformes & Ciconiidae & Ciconia nigra & Zanrai & $\mathrm{R}(\mathrm{C})$ \\
\hline Ciconiiformes & Ciconiidae & Ephippiorhynchus asiaticus & Zanrai & $\mathrm{R}(\mathrm{C})$ \\
\hline Ciconiiformes & Ciconiidae & Ciconia ciconia & Zanrai & $\mathrm{R}(\mathrm{C})$ \\
\hline Columbiformes & Columbidae & Chalcophaps indica & Toti ranga kautara & $\mathrm{M}(\mathrm{SM})(\mathrm{r})$ \\
\hline Columbiformes & Columbidae & Streptopelia decaocto & Kautara & $\mathrm{M}(\mathrm{SM})(\mathrm{C})$ \\
\hline Columbiformes & Columbidae & Columba livia & Shna Kautara & $\mathrm{M}(\mathrm{SM})(\mathrm{C})$ \\
\hline Columbiformes & Columbidae & Columba rupestris & Shna Kautara & $M(S M)(C)$ \\
\hline Columbiformes & Columbidae & Columba leuconota & Kautara & $M(S M)(C)$ \\
\hline Columbiformes & Columbidae & Columba eversmanni & Banj karoro kautara & $\mathrm{M}(\mathrm{SM})(\mathrm{C})$ \\
\hline Columbiformes & Columbidae & Columba palumbus & Shna Kautara & $\mathrm{M}(\mathrm{SM})(\mathrm{C})$ \\
\hline Columbiformes & Columbidae & Columba hodgsonii & Tapasai kautara & $\mathrm{M}(\mathrm{SM})(\mathrm{C})$ \\
\hline Columbiformes & Columbidae & Streptopelia turtur & Kanra kautara & $\mathrm{M}(\mathrm{SM})(\mathrm{C})$ \\
\hline Columbiformes & Columbidae & Streptopelia chinensis & Kautara & $\mathrm{M}(\mathrm{SM})(\mathrm{C})$ \\
\hline Columbiformes & Columbidae & Streptopelia senegalensis & Spalama kautara & $\mathrm{M}(\mathrm{SM})(\mathrm{C})$ \\
\hline Columbiformes & Columbidae & Treron pompadora & Toti ranga kautara & $\mathrm{M}(\mathrm{SM})(\mathrm{r})$ \\
\hline Columbiformes & Columbidae & Treron phoenicoptera & Toti ranga kautara & $\mathrm{M}(\mathrm{SM})(\mathrm{r})$ \\
\hline Columbiformes & Columbidae & Streptopelia orientalis & Karkorai kautara & $\mathrm{M}(\mathrm{SM})(\mathrm{C})$ \\
\hline Coraciiformes & Alcedinidae & Halcyon pileata & Shentagh & $\mathrm{R}(\mathrm{C})$ \\
\hline Coraciiformes & Alcedinidae & Alcedo atthis & Shentagh & $\mathrm{R}(\mathrm{C})$ \\
\hline Coraciiformes & Alcedinidae & Megaceryle lugubris & Mula chargakh & $\mathrm{R}(\mathrm{C})$ \\
\hline Coraciiformes & Coraciidae & Coracias garrulus & Shentagh & $\mathrm{R}(\mathrm{C})$ \\
\hline Coraciiformes & Upupidae & Upupa ерорs & Mula chargakh & $\mathrm{M}(\mathrm{r})$ \\
\hline Galliformes & Phasianidae & Alectoris chukar & Zarka & $\mathrm{R}(\mathrm{C})$ \\
\hline Galliformes & Phasianidae & Francolinus francolinus & Taro & $\mathrm{R}(\mathrm{C})$ \\
\hline Galliformes & Phasianidae & Francolinus pondicerianus & Tanzarai & $\mathrm{R}(\mathrm{C})$ \\
\hline Galliformes & Phasianidae & Coturnix coturnix & Batair & $\mathrm{R}(\mathrm{C})$ \\
\hline Galliformes & Phasianidae & Coturnix coromandelica & Batair & $\mathrm{R}(\mathrm{C})$ \\
\hline Galliformes & Phasianidae & Perdicula asiatica & Batair & $\mathrm{R}(\mathrm{C})$ \\
\hline Galliformes & Phasianidae & Lophophorus impejanus & Late & $\mathrm{R}(\mathrm{C})$ \\
\hline Galliformes & Phasianidae & Catreus wallichii & Sham & $\mathrm{R}(\mathrm{C})$ \\
\hline Galliformes & Phasianidae & Lophura leucomelanos & Taro & $\mathrm{R}(\mathrm{C})$ \\
\hline Gruiformes & Turnicidae & Turnix suscitator & Nwaraz & $\mathrm{R}(\mathrm{C})$ \\
\hline Gruiformes & Turnicidae & Turnix sylvatica & Nwaraz & $\mathrm{R}(\mathrm{C})$ \\
\hline Gruiformes & Gruidae & Grus antigone & Deng & $\mathrm{M}(\mathrm{C})$ \\
\hline Gruiformes & Gruidae & Grus nigricollis & Deng & $\mathrm{M}(\mathrm{C})$ \\
\hline Gruiformes & Gruidae & Anthropoides virgo & Deng & $\mathrm{M}(\mathrm{C})$ \\
\hline Gruiformes & Rallidae & Gallicrex cinerea & Khwar chargai & $\mathrm{R}(\mathrm{C})$ \\
\hline Gruiformes & Rallidae & Gallinula chloropus & Khwar chargai & $\mathrm{R}(\mathrm{C})$ \\
\hline Passeriformes & Sturnidae & Sturnus vulgaris & Sakhakha & $\mathrm{M}(\mathrm{WM})(\mathrm{C})$ \\
\hline Passeriformes & Sturnidae & Acridotheres ginginianus & Kharoo & $\mathrm{R}(\mathrm{C})$ \\
\hline Passeriformes & Sturnidae & Acridotheres tristis & Kharoo & $\mathrm{R}(\mathrm{C})$ \\
\hline
\end{tabular}


TABle 1: Continued.

\begin{tabular}{|c|c|c|c|c|}
\hline Order & Family & Scientific name & Local name & Status \\
\hline Passeriformes & Zosteropidae & Zosterops palpebrosus & Zyar chatai & $\mathrm{R}(\mathrm{C})$ \\
\hline Passeriformes & Dicaeidae & Dicaeum erythrorhynchos & Chatai & $\mathrm{R}(\mathrm{C})$ \\
\hline Passeriformes & Passeridae & Passer domesticus & Chanchanra & $\mathrm{R}(\mathrm{C})$ \\
\hline Passeriformes & Corvidae & Corvus splendens & Kargha & $\mathrm{R}(\mathrm{C})$ \\
\hline Passeriformes & Corvidae & Corvus corone & Kagha & $\mathrm{R}(\mathrm{C})$ \\
\hline Passeriformes & Leiothrichidae & Turdoides caudata & Soorra & $\mathrm{R}(\mathrm{C})$ \\
\hline Passeriformes & Hirundinidae & Hirundo rustica & Totakarkai & $\mathrm{M}(\mathrm{WM})(\mathrm{C})$ \\
\hline Passeriformes & Dicruridae & Dicrurus macrocercus & Toranakha & $\mathrm{M}(\mathrm{SM})(\mathrm{C})$ \\
\hline Passeriformes & Monarchidae & Terpsiphone paradise & Partoghakhai & $\mathrm{M}(\mathrm{WM})(\mathrm{C})$ \\
\hline Passeriformes & Certhiidae & Certhia himalayana & Tak takai & $\mathrm{R}(\mathrm{r})$ \\
\hline Passeriformes & Ploceidae & Ploceus philippinus & Tan tanai & $\mathrm{R}(\mathrm{C})$ \\
\hline Passeriformes & Laniidae & Lanius vittatus & Teghak & $\mathrm{R}(\mathrm{r})$ \\
\hline Passeriformes & Fringillidae & Carpodacus pulcherrimus & Sur sare & $\mathrm{M}(\mathrm{SM})(\mathrm{C})$ \\
\hline Passeriformes & Pycnonotidae & Pycnonotus atriceps & Balbala & $\mathrm{R}(\mathrm{C})$ \\
\hline Passeriformes & Pycnonotidae & Pycnonotus leucogenys & Balbala & $\mathrm{R}(\mathrm{C})$ \\
\hline Passeriformes & Pycnonotidae & Pycnonotus leucotis & Balbala & $\mathrm{R}(\mathrm{C})$ \\
\hline Passeriformes & Pycnonotidae & Pycnonotus cafer & Balbala & $\mathrm{R}(\mathrm{C})$ \\
\hline Passeriformes & Emberizidae & Emberiza melanocephala & Tan tanai & $\mathrm{R}(\mathrm{C})$ \\
\hline Passeriformes & Emberizidae & Emberiza fucata & Chanchanra & $\mathrm{R}(\mathrm{C})$ \\
\hline Passeriformes & Emberizidae & Melophus lathami & Tan tanai & $\mathrm{R}(\mathrm{C})$ \\
\hline Passeriformes & Motacillidae & Motacilla alba & Sper lakai & $\mathrm{R}(\mathrm{C})$ \\
\hline Passeriformes & Motacillidae & Motacilla madaraspatensis & Sper lakai & $\mathrm{R}(\mathrm{C})$ \\
\hline Passeriformes & Motacillidae & Motacilla citreola & Sper lakai & $\mathrm{R}(\mathrm{C})$ \\
\hline Passeriformes & Motacillidae & Motacilla lugens & Sper lakai & $\mathrm{R}(\mathrm{C})$ \\
\hline Passeriformes & Campephagidae & Pericrocotus erythropygius & Tan tanai & $\mathrm{R}(\mathrm{C})$ \\
\hline Passeriformes & Regulidae & Regulus regulus & Tan tanai & $\mathrm{R}(\mathrm{C})$ \\
\hline Passeriformes & Aegithinidae & Aegithina tiphia & Tan tanai & $\mathrm{R}(\mathrm{C})$ \\
\hline Passeriformes & Cinclidae & Cinclus pallasii & Dabagai & $\mathrm{R}(\mathrm{C})$ \\
\hline Pelecaniformes & Phaethontidae & Phaethon aethereus & Batha & $\mathrm{M}(\mathrm{SM})(\mathrm{C})$ \\
\hline Pelecaniformes & Pelecanidae & Pelecanus onocrotalus & Batha & $\mathrm{M}(\mathrm{SM})(\mathrm{C})$ \\
\hline Pelecaniformes & Pelecanidae & Pelecanus philippensis & Batha & $\mathrm{M}(\mathrm{SM})(\mathrm{C})$ \\
\hline Pelecaniformes & Pelecanidae & Pelecanus crispus & Batha & $\mathrm{M}(\mathrm{SM})(\mathrm{C})$ \\
\hline Pelecaniformes & Anhingidae & Anhinga melanogaster & Batha & $\mathrm{M}(\mathrm{SM})(\mathrm{C})$ \\
\hline Phoenicopteriformes & Phoenicopteridae & Phoenicopterus roseus & Deng & $\mathrm{M}(\mathrm{SM})(\mathrm{r})$ \\
\hline Phoenicopteriformes & Phoenicopteridae & Phoenicopterus minor & Deng & $\mathrm{M}(\mathrm{SM})(\mathrm{r})$ \\
\hline Piciformes & Picidae & Dendrocopos moluccensis & Tak takai & $\mathrm{R}(\mathrm{r})$ \\
\hline Psittaciformes & Psittacidae & Psittacula krameri & Toti & $\mathrm{M}(\mathrm{C})$ \\
\hline Psittaciformes & Psittacidae & Psittacula himalayana & Toti & $\mathrm{M}(\mathrm{C})$ \\
\hline Psittaciformes & Psittacidae & Psittacula cyanocephala & Toti & $\mathrm{M}(\mathrm{C})$ \\
\hline
\end{tabular}

\section{Discussion}

The food availability, feeding, and habitats may be the main factors of variation in the birds population slightly than any other risk [20]. In our study it was found that feeding and habitat availability play important roles in the diversity and distribution of the avian fauna of area. The flora of the study area was rich and due to the thick flora the study area was bearing rich avian fauna.

The birds are intensively hunted and captured in their native range in Pakistan, owing to which local populations could be declining, but the overall status of the species is regarded as stable [12-14]. In our study it was observed that the birds were hunted but the hunting ratio was low due to current situation of terrorism in Swat; therefore the bird fauna was rich.

To avoid the severe winter season a large number of birds migrate from central Asian countries and Europe towards wetlands of Pakistan. There are seven fly zones all over the world in which one zone (Indus fly zone) is present in Pakistan. The birds reach Pakistan flying over Karakorum, Sulaiman Ranges, and Hindu Kush along the Indus River. 
Falcons, cranes, swans, ducks, flamingos, waders, and geese are important migratory birds in host country [21]. Similarly in our study the migratory birds recorded were ducks, geese, and swans, which were mostly summer visitors.

Birds are normally found foraging in open cultivated tracks and grasslands intermixed with scrub forests and are rarely observed above an elevation of $1200 \mathrm{~m}$ in Pakistan [22]. The elevation of the study area ranges from 4500 to over 6000 meters.

Rose ringed parakeet, house crow, house sparrow, mynas, and bulbuls were common among the resident birds, while kingfisher, koel, rollers, and tree pie were rare in number and have small spread families [23]. House sparrow, house crow, myna, and bulbul were recorded as residential and abundant as also reported previously $[24,25]$. In our study the resident birds were chukars, pheasants, house crow, house sparrow, mynas, and bulbuls which are quite similar to the previous studies.

Common sandpiper is common winter visitor to Azad Kashmir. Plumbeous redstart and river chats are also common and locally migrant [25]. Similarly in our study sandpipers were found as winter visitors.

Alectoris chukar has worldwide distribution, which is found in India, Afghanistan, Middle East, and western Himalayas, east to central Nepal [6]. In Pakistan, Alectoris chukar is very adaptable to all kinds of the arid, rocky, and hilly country ascending to the higher mountain valleys of the inner Himalayas ranges [15] and bare, arid hillside of the Punjab and western Himalayas [26]. It is distributed throughout Pakistan in certain places, that is, Punjab, Sind, Baluchistan, Chitral, Salt range, Swat, Kohistan, and Gilgit $[15,26]$. This bird is also found throughout the AJK [27]. In our study the Alectoris chukar was found widely in many numbers due to less hunting in the study area due to the cease fire in the Swat.

The Grey Francolin (Francolinus pondicerianus) is widely associated with the drier regions of the Indus plains and has penetrated the Thar Desert in Sindh, as well as the Thal and Cholistan deserts in Punjab. The species also appears in the lower hills of the Makran and Lasbela districts in Balochistan, the Cherat and Kohat districts of Khyber Pakhtunkhwa province, the salt range and agroforestry tracks of the Pothwar Plateau in the Punjab, and the Margalla hills of Islamabad $[17,18]$. In our study the Grey Francolin was found in many numbers.

The Quail carries out all its vital functions (feeding and nest-building) in the herbaceous strata of natural coastal grasslands (abundant grasses), high altitude prairies (e.g., uncultivated land in the Aveyron and Capcir, France) or, as for the Grey Partridge Perdix perdix, the grassy areas of open agrosystems (with the notable exception of ryegrass). The Quail prefers cover which, although dense enough to provide protection, allows fluid movements, hence the choice of alfalfa, winter barley, and winter wheat when still green and showing abundant basal leaves or early shoots [28]. As the flora of the study area was very thick, the Quails and Grey Partridge were found in large numbers as there were many places of shelters for their breeding and other activities.
Red Turtle Dove is summer visitor and spotted dove is common [15]. The work of [25] reported its status as common. This species is found in Himalaya and Kashmir but locally migrant [24]. In our study the Rock Pigeon, Hill Pigeon, Snow Pigeon, Pale-backed Pigeon, Common Wood-Pigeon, Speckled Wood-Pigeon, Eurasian Turtle Dove, and other members of the same family were recorded as migratory and were found to be summer visitors.

\section{Conclusion}

Hunting and habitat destruction are major threats to wildlife. Fauna of an area depends on the flora present in the area because it provides food and shelter to the fauna and destruction of the habitats also results in the elimination or migration of species. The avian fauna of the study area was rich because the flora was thick. Hunting in the study area was very much loss due to the cease fire in Swat due to the current situation of terrorism in Swat. It was concluded from the current study that hunting and habitat destruction are the major threats to the wildlife.

\section{Conflict of Interests}

The authors declare that there is no conflict of interests regarding the publication of this paper.

\section{Acknowledgment}

The authors are very thankful to the local villagers and hunters who helped them during their field work and also helped them in the identification of birds by naming them by their local names.

\section{References}

[1] M. A. Beg and J. J. Qureshi, "Birds and their habitats in the cultivated areas of Lyallpur district and vicinity," Pakistan Journal of Agricultural Sciences, vol. 9, pp. 161-166, 1972.

[2] A. Mian, "Grey partridge demands intensive study," WPA News, vol. 1, no. 2, 1995.

[3] M. Chittampalli and B. P. N. Bhatkhande, Hansdev's Mrigpakeshi Shastra, M.S. Board of Literature and Culture, Mumbai, India, 1993.

[4] H. Kafley, Habitat Evaluation and Suitability Modeling of Rhinoceros unicornis in Chitwan National Park, A Geospatial Approach, Institute of International Education World Wildlife Fund ITTO, Kathmandu, Nepal, 2008.

[5] R. L. Smith, Ecology and Field Biology, Harper and Row, New York, NY, USA, 1974.

[6] S. Ali and S. D. Ripley, Hand Book of the Birds of India and Pakistan, Oxford University Press, 1987.

[7] R. D. Gregory, D. Noble, R. Field, J. Marchant, M. Raven, and D. W. Gibbons, "Using birds as indicators of biodiversity," Ornis Hungarica, vol. 12-13, pp. 11-24, 2003.

[8] R. Grimmett, C. Inskipp, and T. Inskipp, Birds of the Indian Subcontinent, Christopher Helm, London, UK, 1998.

[9] A. A. Khan, R. Khan, A. Ullah, M. Ali, J. A. Mahmood, and K. M. Sheikh, "Conservation perspectives of the imperial Aquila 
heliaca and steppe eagle Aquila nipalensis in Pakistan," in Eagle Studies World Walking Group on Birds of Prey (WWGBP), B.-U. Meyburg and R. D. Chancellor, Eds., 1996.

[10] R. Grimmett, C. Inskipp, and T. Inskipp, Birds of Indian Subcontinent, Christopher Helm, London, UK, 2001.

[11] Z. B. Mirza and H. Wasiq, A Field Guide to Birds of Pakistan, WWF-Pakistan, Bookland Lahore, 2007.

[12] J. del Hoyo, A. Elliott, and J. Sargatal, Handbook of the Birds of the World. Volume 2. New World Vultures to Guinea Fowl, Lynx Edicions, Barcelona, Spain, 1994.

[13] K. Islam, "Erckel's francolin (Francolinus erckelii), black fran colin (Francolinus francolinus), and grey francolin (Francolinus pondicerianus)," in The Birds of North America, A. Poole and F. Gill, Eds., pp. 394-396, Birds of North America Online, Philadelphia, Pa, USA, 1999.

[14] A. F. Richard, J. P. Caroll, and J. P. K. McGown, Partridges, Quails, Francolins, Snowcocks, Guineafowl and Turkeys, IUCN, The World Conservation Union, Gland, Switzerland, 2002.

[15] I. Newton, "The contribution of some recent research on birds to ecological understanding," Journal of Animal Ecology, vol. 64, no. 6, pp. 675-696, 1995.

[16] T. J. Roberts, The Birds of Pakistan: Non-Passeriformes, Oxford University Press, New York, NY, USA, 1991.

[17] K. Mahmood, T. Ahmad, A. Khan, A. Mahmood, and W. Mahmood, "Some notes on avifauna of Mangla reservoir, AJK, Pakistan," Pakistan Journal of Ornithology, vol. 1, no. 1, pp. 31-37, 1997.

[18] M. A. Mann and A. Chaudhry, "Francolins in irrigated forest plantations and sub-mountainous tract of the Punjab, Pakistan," The Pakistan Veterinary Journal, vol. 20, no. 3, pp. 118-122, 2000.

[19] Wildlife Department, Khyber Pakhtunkhawa, 2008.

[20] Z. Ali, F. Bibi, S. Y. Shelly, A. Qazi, and A. M. Khan, "Comparative avian faunal diversity of Jiwani Coastal Wetlands and Taunsa barrage Wildlife Sanctuary, Pakistan," Journal of Animal and Plant Sciences, vol. 21, no. 2, pp. 381-387, 2011.

[21] Z. Ali and M. Akhtar, "Bird surveys at wetlands in Punjab, Pakistan, with special reference to the present status of Whiteheaded Duck Oxyura leucocephala," Forktail, vol. 21, pp. 43-50, 2005.

[22] R. Grimmett, T. J. Roberts, and T. Inskipp, Birds of Pakistan, Yale University Press, 2008.

[23] M. N. Awan, M. S. Awan, K. B. Ahmed, A. A. Khan, and N. I. Dar, "A preliminary study on distribution of avian fauna of muzaffarabad-azad Jammu and Kashmir, Pakistan," International Journal of Agriculture and Biology, vol. 6, no. 2, 2004.

[24] H. Whistler, A Popular Handbook of Indian Birds, Oxford University Press, Bombay, India, 1949.

[25] T. J. Roberts, The Birds of Pakistan Vol. 2, Passerriformes, Oxford University Press, Oxford, UK, 1992.

[26] M. Wood Cock, Collins Birds of the Indian Sub-Continent, Harper Collins, London, UK, 1980.

[27] Anonymous, "Wildlife Survey Report," Wildlife wing, Forest department Azad government of the State of Jammu and Kashmir, 1985.

[28] Aubraiso, Y. A. Hemony, and J. C. Guyomarch, "Habitat et occupation de l'espace chez la caille des blés (Coturnix coturnix) au début de la période de reproduction," Gibier, Faune Sauvage, vol. 3, pp. 317-342, 1986. 

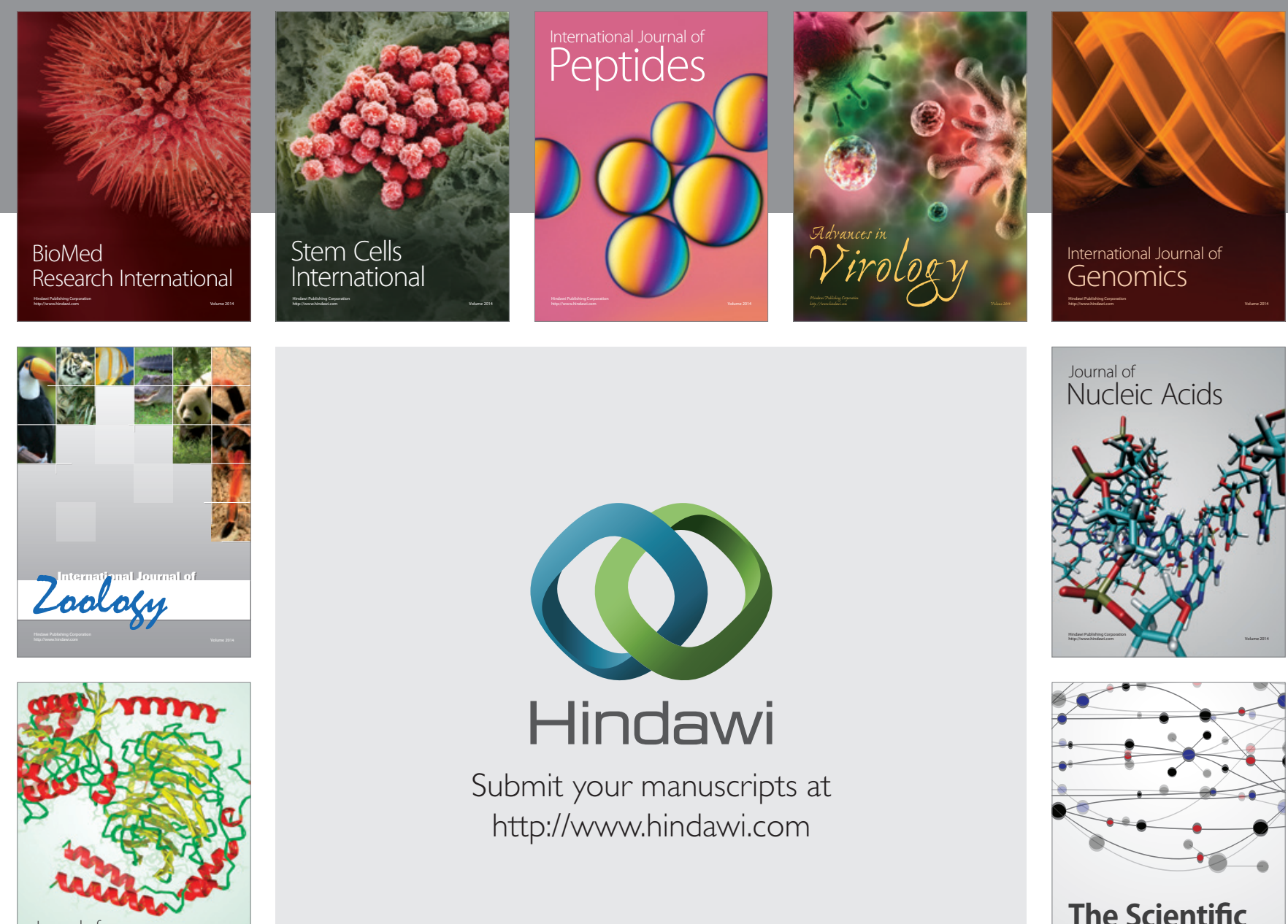

Submit your manuscripts at

http://www.hindawi.com

Journal of
Signal Transduction
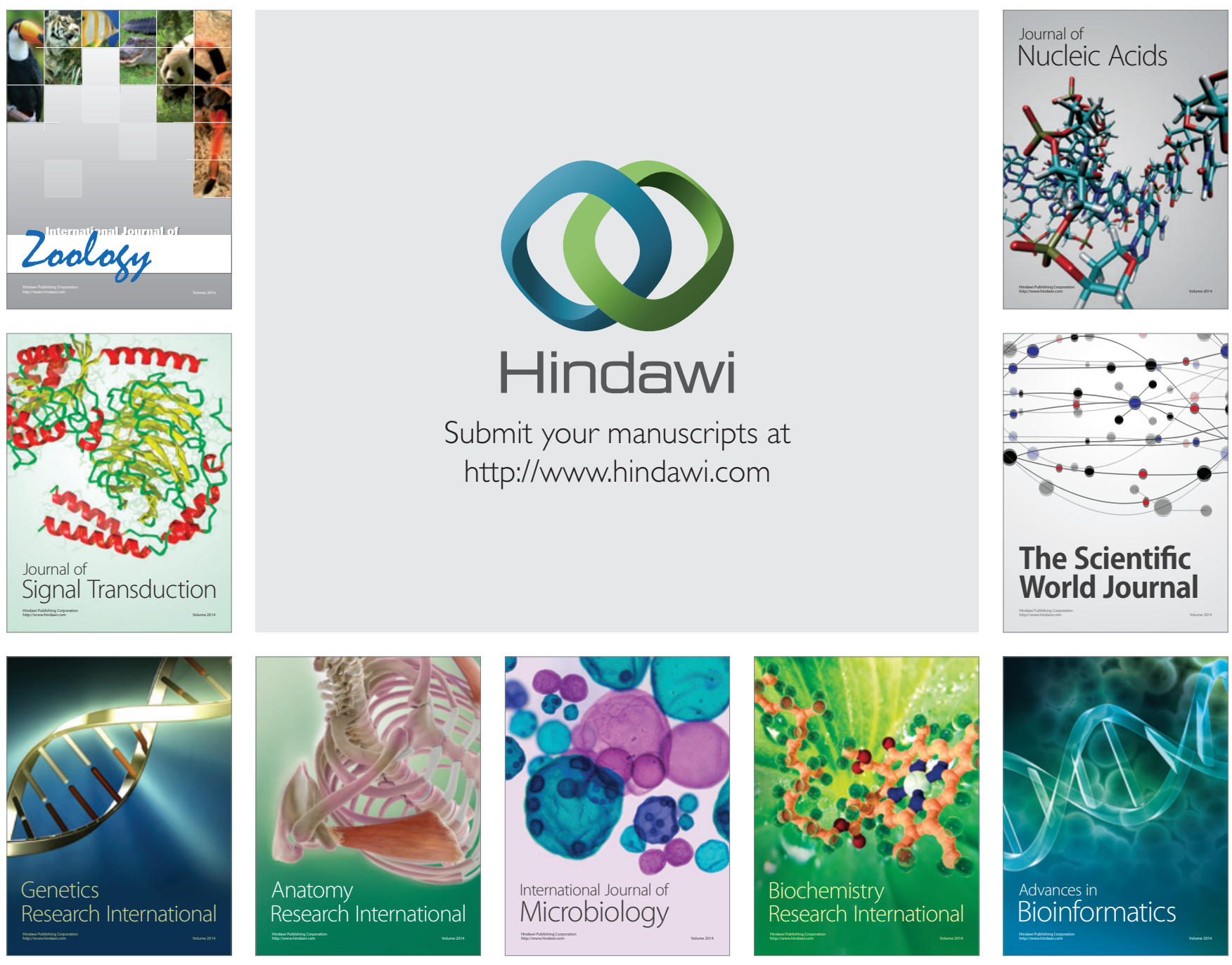

The Scientific World Journal
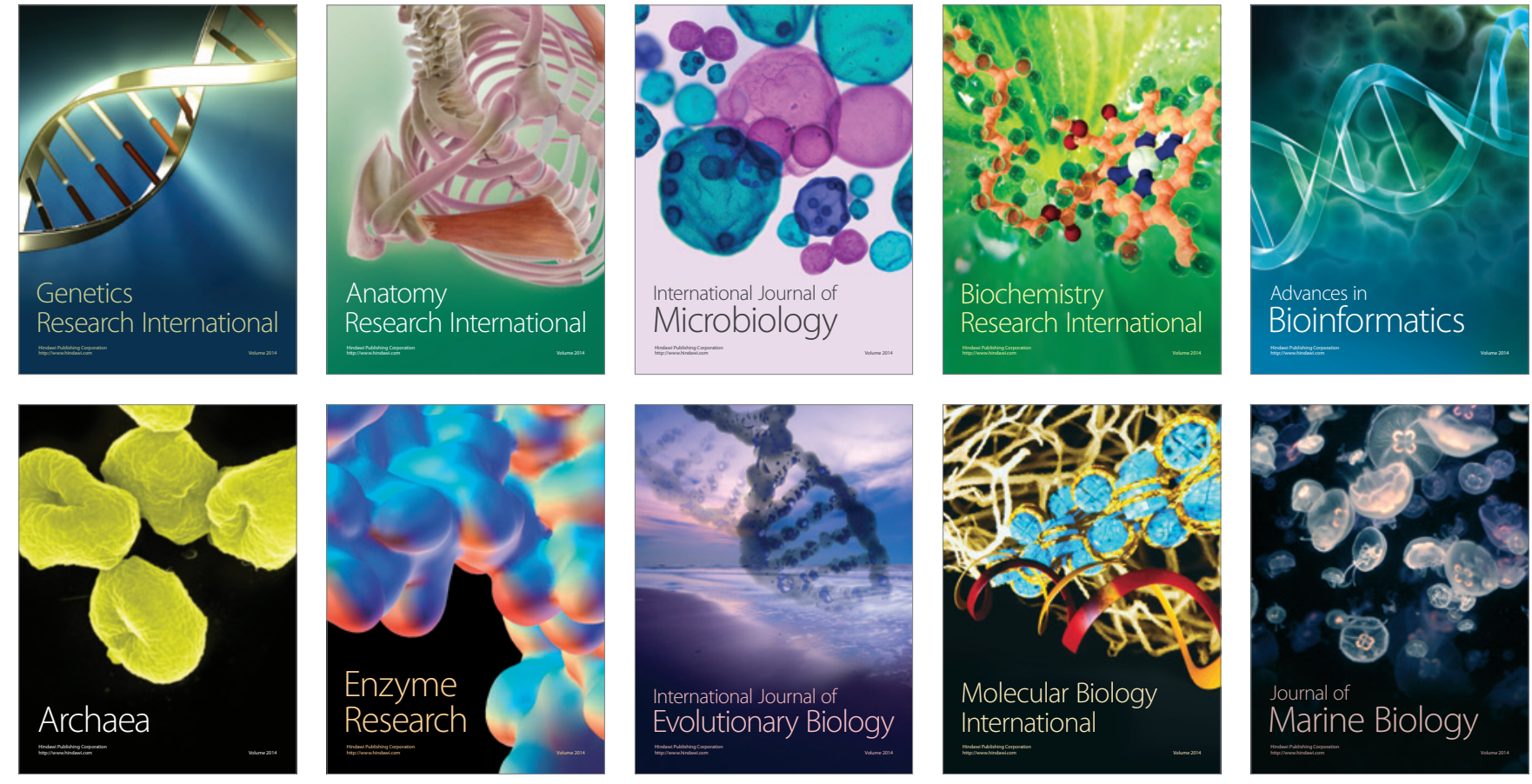